\title{
Cerebrospinal fluid soluble TREM2 in aging and Alzheimer's disease
}

Kristi Henjum ${ }^{1}$, Ina S. Almdah2 ${ }^{2,3 \dagger}$, Vibeke Årskog ${ }^{1 \dagger}$, Lennart Minthon ${ }^{4}$, Oskar Hansson ${ }^{4}$, Tormod Fladby 2,3

and Lars N. G. Nilsson ${ }^{1 *}$

\begin{abstract}
Background: Alzheimer's disease (AD) neuropathology is associated with neuroinflammation, but there are few useful biomarkers. Mutant variants of triggering receptor expressed on myeloid cells 2 (TREM2) have recently been linked to late-onset AD and other neurodegenerative disorders. TREM2, a microglial receptor, is involved in innate immunity. A cleaved fragment, soluble TREM2 (STREM2), is present in the cerebrospinal fluid (CSF).
\end{abstract}

Methods: We developed and used a novel enzyme-linked immunosorbent assay to investigate the potential value of CSF sTREM2 as an AD biomarker in two independent cohorts: an AD/mild cognitive impairment (MCl)/control cohort $(n=100)$ and an AD/control cohort $(n=50)$.

Results: We found no significant difference in sTREM2 levels between groups of controls and patients with AD or MCl. However, among all controls there was a positive correlation between STREM2 and age (Spearman rho $=0.50 ; p<0.001$; $n=75)$. In the AD/MCl/control cohort, CSF sTREM2 correlated positively with total Tau (T-tau) (Spearman rho 0.57; $p<0.001 ; n=50)$, phosphorylated Tau (P-tau) (Spearman rho 0.63; $p<0.001 ; n=50$ ) and amyloid- $\beta 1-42$ (Aß42) (Spearman rho $0.35 ; p=0.01 ; n=50$ ) in control subjects. Among controls with a CSF A $\beta 42$ above a cut-off value $(700 \mathrm{pg} / \mathrm{ml})$ in this cohort, the positive correlation between sTREM2 and A 342 was stronger (Spearman rho $=0.44$; $p=0.002 ; n=46)$.

Conclusions: sTREM2 in CSF correlates with aging in controls, and with the neurodegenerative markers CSF T-tau/P-tau among controls who are negative for AD CSF core biomarkers Aß42, T-tau or P-tau.

Keywords: Aging, Alzheimer's disease, Amyloid beta, Microgliosis, Mild cognitive impairment, Neuroinflammation, Soluble TREM2, Tau

\section{Background}

Alzheimer's disease (AD) is characterized by neurodegeneration in the presence of two neuropathological hallmarks, extracellular amyloid-beta $(A \beta)$ plaques and intracellular neurofibrillary tangles (NFTs), in certain brain regions [1]. A definitive $\mathrm{AD}$ diagnosis relies on post-mortem histological confirmation, but imaging and analyses of cerebrospinal fluid (CSF) A $\beta 1-42$ (A 342$)$, total Tau (T-tau) and phosphorylated Tau (P-tau) have transformed the field by enabling early and reliable ante-mortem $\mathrm{AD}$ diagnosis. These biomarkers are now included in the diagnostic guidelines [2-4], but additional AD biomarkers are needed

\footnotetext{
* Correspondence: lars.nilsson@medisin.uio.no

${ }^{\dagger}$ Equal contributors

${ }^{1}$ Department of Pharmacology, University of Oslo and Oslo University

Hospital, P.b. 1057, Blindern NO-0316, Oslo, Norway

Full list of author information is available at the end of the article
}

to measure specific aspects and progression of this complex disease. The AD brain is also affected by neuroinflammation, but the immune reactions involved are intricate and their role in pathogenesis is partly unclear. Moreover biomarkers of neuroinflammation are scarce [5]. Longitudinal monitoring of astroglial or microglial activation with positron emission tomography (PET) imaging is used in research but not established in clinical practice [6]. In CSF, the potential value of many immune mediators as biomarkers has been examined. A diagnostic or prognostic value of different complement factors, acute-phase proteins, cytokines or chemokines has been reported, but findings have seldom been robust and reproducible [7]. YKL-40 might become a clinically useful marker of astrogliosis [8], but markers of microgliosis will also be needed. 
Multiple genetic studies of late-onset $\mathrm{AD}$ point to a causative role of innate immunity in the $\mathrm{AD}$ pathogenesis [9-11]. Triggering receptor expressed on myeloid cells 2 (TREM2) is expressed abundantly by microglia [12,13] and TREM2 is a susceptibility gene for late-onset AD [14, 15]. Gene-expression analyses of late-onset AD post-mortem brain also suggest that an immune-specific and microgliaspecific module around the TREM2 signalling adapter DNAX activating protein 12 (DAP12) is involved in the pathogenesis [16]. Moreover, TREM2 expression is elevated with aging in human brain [12] and in the vicinity of amyloid deposits in transgenic mouse models of AD [17]. TREM2 is a transmembrane innate immune receptor undergoing ectodomain cleavage with extracellular release of a soluble TREM2 (sTREM2) fragment which is detectable in CSF $[18,19]$. A disintegrin and metalloproteinase (ADAM)-10, a key enzyme for $\alpha$-secretase cleavage of $\mathrm{A} \beta$ precursor protein $(\mathrm{A} \beta \mathrm{PP})$, cleaves the TREM2 ectodomain [18]. The remaining TREM2 C-terminal fragment is digested by $\gamma$-secretase [20]. Since both genetic and pathological studies link TREM2 to AD, sTREM2 might be a useful biomarker of microglial activation or neurodegeneration. Improved abilities to monitor microglial function and activity would also facilitate development of new microglial-based therapeutics. In the present study, we developed and validated an enzyme-linked immunosorbent assay (ELISA) and explored whether sTREM2 could serve as a diagnostic biomarker for $\mathrm{AD}$ or mild cognitive impairment (MCI). Moreover, we examined whether sTREM2 levels correlated with the established AD CSF core biomarkers A 342 , T-tau or P-tau. We also analysed the effect of normal aging, the most important risk factor of AD.

\section{Methods}

\section{Clinical samples}

The Swedish cohort was from the Memory Clinic of Skåne University Hospital in Malmö, Sweden, and comprised 25 patients diagnosed with $\mathrm{AD}$ and 25 non-AD individuals (controls). Patients diagnosed with AD met the DSM-IIIR criteria for dementia [21] and the criteria for probable $\mathrm{AD}$, as defined by the National Institute of Neurological and Communicative Disorders and Stroke (NINCDS-ADRDA) [22]. All subjects were carefully assessed and tested by medical doctors with extensive experience in cognitive disorders. Their brains were examined with either magnetic resonance imaging (MRI) or computed tomography (CT). Controls were clinically followed up to ensure that the cognitive complaints at baseline were not due to dementia or any other neurodegenerative disorder. The CSF samples of all patients were collected as part of routine clinical investigation. In conjunction with the investigation, oral informed consent for future use of their banked CSF samples for research purposes was obtained and documented in the patients' medical records. All patients were later instructed to withdraw their permission if they changed their minds, as advertised in the local press. The design of the study was approved by the Local Ethics Committee of Lund University in Sweden (permit 2010-401), and the study procedure was conducted in accordance with the Declaration of Helsinki.

The Norwegian cohort was from the Memory Clinic of Akershus University Hospital in Lørenskog, Norway. The cohort encompassed 50 patients diagnosed with either AD or $\mathrm{MCI}$, due to a pre-dementia stage of $\mathrm{AD}$, and 50 cognitively healthy controls. All patients were interviewed and examined by a physician trained in diagnosing cognitive disorders. They all underwent cognitive testing, either cerebral MRI or CT, blood screening and standard lumbar puncture as part of the clinical assessment. Patients met either the National Institute on Aging-Alzheimer's Association (NIA-AA) criteria for dementia due to $\mathrm{AD}$ [4] or the high-likelihood NIA-AA criteria for MCI due to AD [2] (29 patients and 21 patients, respectively). The controls were either orthopaedic patients scheduled for elective joint replacement surgery, spouses of patients attending the Memory Clinic or individuals recruited through newspaper advertisement. CSF was collected before administration of spinal anaesthesia in the orthopaedic patients. The remaining controls underwent standard lumbar puncture. Inclusion criteria for the controls were the absence of any reported cognitive complaints and normal CSF A $\beta 42$ concentrations according to the cut-off value set by the laboratory (>550 pg/ml, modified from [23, 24]). All controls were invited to undergo further assessments; 35 consented to cognitive testing and 32 to cerebral MRI. Exclusion criteria for both AD patients and controls were: any ongoing severe neurological, medical or psychiatric co-morbidity or treatment with the potential to impair cognitive functioning; systemic inflammatory disease or infection based on clinical and laboratory assessment; and use of immunosuppressant drugs. The three groups were matched for age and gender. The Regional Committee for Medical and Health Research Ethics, South East Norway, approved the study (approval 1.2007.2511, 2011/1015 and 2013/150) and all participants gave written informed consent. The study procedure was conducted in accordance with the Declaration of Helsinki. The demographics and clinical characteristics of both cohorts are presented in Table 1.

The TREM2 [p. T66M] mutation prevents shedding of TREM2, and CSF from a patient homozygous for [p. T66M] is reported to be devoid of sTREM2 [18]. A sample from such a patient was used to verify that the ELISA signal was specific for sTREM2, and not partly due to other components in the CSF matrix. Genomic DNA from the $[\mathrm{p} . \mathrm{T} 66 \mathrm{M}]$ mutation carrier had been analysed by exome sequencing of the entire genome [25]. 
Table 1 Characteristics of the Norwegian and Swedish cohorts

\begin{tabular}{|c|c|c|c|c|c|c|}
\hline & & & & $p$ value & & \\
\hline & Controls & $\mathrm{MCl}$ & $A D$ & Controls-MCl & Controls-AD & $\mathrm{MCl}-\mathrm{AD}$ \\
\hline Norwegian cohort & $n=50$ & $n=21$ & $n=29$ & & & \\
\hline Gender & & & & & & \\
\hline Women & 25 & 12 & 13 & & & \\
\hline Men & 25 & 9 & 16 & & & \\
\hline Age & $66(50-86)$ & $67(55-75)$ & $68(56-75)$ & & & \\
\hline MMSE & $29(29-30)$ & $27(26-29)$ & $20(17-24)$ & * & * & $*$ \\
\hline CSF Aß42 (pg/ml) & $1010(880-1188)$ & $494(356-531)$ & $500(386-553)$ & * & $*$ & 0.43 \\
\hline CSF T-tau (pg/ml) & $307(201-391)$ & $628(497-927)$ & $772(647-1143)$ & * & * & 0.06 \\
\hline CSF P-tau (pg/ml) & $51(38-61)$ & $75(62-111)$ & 72 (67-91) & * & * & 1.00 \\
\hline ApoE genotype & & & & & & \\
\hline E2/E3 & 8 & - & 1 & & & \\
\hline E2/E4 & - & & 1 & & & \\
\hline E3/E3 & 35 & 5 & 5 & & & \\
\hline $\mathrm{E} 3 / \mathrm{E} 4$ & 6 & 8 & 12 & & & \\
\hline E4/E4 & - & 8 & 10 & & & \\
\hline Not known & 1 & & & & & \\
\hline CSF sTREM2 (ng/ml) & $4.4(3.0-5.7)$ & $4.1(2.4-5.9)$ & $4.8(3.5-7.1)$ & 0.42 & 0.17 & 0.11 \\
\hline Swedish cohort & $n=25$ & & $n=25$ & & & \\
\hline Gender & & & & & & \\
\hline Women & 17 & & 18 & & & \\
\hline Men & 8 & & 7 & & & \\
\hline Age & $62(43-80)$ & & $79(61-86)$ & & & \\
\hline MMSE & $29(28-30)$ & & $18(13-22)$ & & * & \\
\hline CSF A $422(\mathrm{pg} / \mathrm{ml})$ & $520(469-597)$ & & $340(265-430)$ & & $*$ & \\
\hline CSF T-tau (pg/ml) & $380(233-480)$ & & $670(490-895)$ & & * & \\
\hline CSF P-tau (pg/ml) & $44(36-61)$ & & $80(69-96)$ & & * & \\
\hline ApoE genotype & & & & & & \\
\hline E2/E3 & 5 & & 1 & & & \\
\hline E2/E4 & 1 & & 1 & & & \\
\hline E3/E3 & 14 & & 4 & & & \\
\hline E3/E4 & 4 & & 15 & & & \\
\hline E4/E4 & 1 & & 4 & & & \\
\hline CSF sTREM2 (ng/ml) & $3.2(2.8-5.0)$ & & $3.8(2.6-5.6)$ & & 0.76 & \\
\hline
\end{tabular}

Descriptive statistics of gender, age, MMSE, ApoE genotype and CSF biomarker data of AD, MCl and control patients of the two cohorts Data are presented as median (interquartile range), except age which is presented as median (minimum-maximum). Two-tailed $p$ values obtained by Mann-Whitney $U$ test for group comparison

${ }^{*} p<0.001$

A 42 amyloid beta 1-42, AD Alzheimer's disease, ApoE apolipoprotein E, CSF cerebrospinal fluid, MCI mild cognitive impairment, MMSE Mini-Mental State Examination, $P$-tau phosphorylated Tau, sTREM2 soluble triggering receptor expressed on myeloid cells 2, T-tau total Tau

\section{CSF collection and storage}

Lumbar puncture was in general performed between 9 a.m. and 12 p.m., predominantly in the L3/L4 or L4/ L5 inter-space and without any reported serious adverse effects. CSF was collected in polypropylene tubes, centrifuged and stored at $-80{ }^{\circ} \mathrm{C}$. The samples were subjected to a maximum of two freeze-thaw cycles prior to determination of sTREM2 levels.

\section{Cell cultures}

The Chinese hamster ovary $\mathrm{CHO}-\mathrm{K} 1$ and THP-1 cell lines were purchased from American Type Culture Collection 
(ATCC, Rockville, MD, USA). CHO-K1 cells were cultured in Dulbecco's modified Eagle medium (DMEM) supplemented with $10 \%$ fetal bovine serum (FBS) and $1 \%$ penicillin-streptomycin (Sigma, St. Louis, MO, USA). These cells were transfected with a synthetic human TREM2DAP12 fusion gene that had been subcloned into the NheI and BgIII sites and thus replaced copGFP in a pmaxGFP expression vector (Lonza, Basel, Switzerland). Transfections were carried out using Lipofectamine LTX and Plus Reagent (Life Technologies, Carlsbad, CA, USA) according to the manufacturer's recommendations. Conditioned cell culture medium (conditioned medium) was collected $15 \mathrm{~h}$ after transfection, centrifuged at $5200 \times g$ for $15 \mathrm{~min}$ at $4{ }^{\circ} \mathrm{C}$ and supplemented with Complete ${ }^{\circ}$ protease inhibitor (Roche, Basel, Switzerland). The cells were washed with PBS and lysed with $0.5 \%(\mathrm{w} / \mathrm{v})$ sodium dodecyl sulphate (SDS) (Sigma) in PBS with Complete ${ }^{\circ}$ and stored at $-20{ }^{\circ} \mathrm{C}$. The THP-1 monocyte cell line was cultured in RMPI with Glutamax (Thermo-Scientific, Waltham, MA, USA) supplemented with $10 \% \mathrm{FBS}$ and $1 \%$ penicillin-streptomycin. For differentiation, $0.1 \mu \mathrm{M}$ phorbol 12-myristate 13-acetate (Sigma) was added to the culture media. The conditioned medium from the differentiated cells was collected after $48 \mathrm{~h}$ and centrifuged at $5200 \times g$ for $7 \mathrm{~min}$. The supernatant was supplemented with Complete, stored at $-80{ }^{\circ} \mathrm{C}$ and used as an internal standard in the TREM2 ELISA.

\section{TREM2 ELISA}

Maxisorp plates (ThermoFischer, Waltham, MA, USA) were coated by shaking them overnight at $4{ }^{\circ} \mathrm{C}$ with capturing goat anti-human TREM2 antibody (R\&D Systems, Minneapolis, MN, USA) at a final concentration of $0.5 \mu \mathrm{g} / \mathrm{ml}$ in $50 \mathrm{mM}$ carbonate-bicarbonate buffer ( $\mathrm{pH}$ 9.6). The plates were then blocked with $2 \%$ $(\mathrm{w} / \mathrm{v})$ bovine serum albumin in TTBS $(20 \mathrm{mM}$ Tris, $150 \mathrm{mM} \mathrm{NaCl}, \mathrm{pH} 7.4$ with $0.05 \%$ (v/v) Tween-20) before sample incubation for $2 \mathrm{~h}$ at room temperature (RT). Samples were diluted (CSF 16x, THP-1 conditioned medium $16 \times$, CHO-KI conditioned medium $50 \times$ and $\mathrm{CHO}-\mathrm{KI}$ cell lysate $200 \times$ ) in TTBS supplemented with $0.1 \%(\mathrm{w} / \mathrm{v})$ bovine serum albumin. The plates were then incubated with HRP-conjugated detecting mouse anti-human TREM2 antibody (Sino Biologics, Beijing, China) at a final concentration of $0.4 \mu \mathrm{g} / \mathrm{ml}$ for $1 \mathrm{~h}$ at RT. TTBS was applied for washing between each step. Finally TMB substrate (ANL produkter, Älvsjö, Sweden) was added for signal detection and development was stopped with $0.2 \mathrm{M} \mathrm{H}_{2} \mathrm{SO}_{4}$ (final concentration). The plates were analysed with a SpectraMAX 190 spectrophotometer at $450 \mathrm{~nm}$ (Molecular Devices, Palo Alto, CA, USA) and the data with SoftMax Pro software (Molecular Devices). Recombinant TREM2 was used as standard (Sino Biologics).

\section{CSF freeze-thaw cycles}

CSF samples $(n=2)$ were subjected to five rounds of thawing and freezing. Briefly, samples of $15 \mu \mathrm{l}$ were thawed for $90 \mathrm{~s}$ in a water bath at room temperature, incubated for $15 \mathrm{~min}$ on ice and then transferred to $-80{ }^{\circ} \mathrm{C}$ for $1 \mathrm{~h}$.

\section{T-tau, P-tau and A 342 ELISAs}

CSF levels of T-tau, P-tau and A $\beta 42$ were quantified with commercially available ELISAs; Innotest ${ }^{\circ} \mathrm{hTau} \mathrm{Ag}$, Innotest $^{\circ}$ phoshoTau (181P) $[26,27]$ and Innotest ${ }^{\circ} \beta$ amyloid 1-42 [28] (Fujirebio Europe, Gent, Belgium). $\mathrm{A} \beta 1-38$ (A $\beta 38), A \beta 1-40(\mathrm{~A} \beta 40)$ and $\mathrm{A} \beta 42$ were analysed using a MSD Multi-Spot Assay System (Meso Scale Discovery, Rockville, MA, USA) with 6E10 (BioLegend, San Diego, CA, USA) as the detection antibody. The Meso Scale Discovery analyses, which were applied to a subset of patients in the Norwegian cohort, were carried out according to the manufacturers' procedures.

\section{Statistical analyses}

The statistical analyses were carried out with the Statistical Package for Social Sciences (SPSS, version 22; IBM, Armonk, NY, USA). Since the majority of the data were skewed, correlations were assessed by non-parametric Spearman rho and differences between groups by MannWhitney $U$ test. Standardized residuals in multiple linear regression analyses gave no indication of violation of the normal distribution. All $p$ values are two-tailed, since all hypotheses tested were two-sided. The significance level was set at 0.05. Graphs were created with GraphPad Prism (version 5.02; Graph Pad Software, La Jolla, CA, USA) or Statistica software (version 10; Statsoft, Uppsala, Sweden).

\section{Results}

Sensitive and specific detection of human TREM2 with a new sandwich ELISA

Human TREM2 cells and mock-transfected CHO-K1 cells served as positive and negative control respectively for ELISA validation. The ELISA easily detected TREM2 in the cell lysate, as well as sTREM2 in the corresponding conditioned medium from TREM2-transfected CHO-K1 cells. The signals from conditioned medium and cell lysate of mock-transfected cells were at the level of the zero standard. Cellular TREM2 and released sTREM2 were thus detectable in complex biological samples without significant cross-reactivity (Fig. 1). Intra-day and inter-day variability was $6.7 \pm 1.7 \%$ and $5.7 \pm 0.3 \%$ respectively. The readings did not depend on dilution of the CSF samples (Additional file 1: Figure S1). The [p. T66M] mutation in TREM2 markedly reduced cell surface transport and shedding of TREM2 [18]. A CSF sample from a patient homozygous for TREM2 [p. T66M] was used to further ensure that the ELISA signal was solely due to STREM2 in CSF. This CSF sample gave an ELISA signal very close to the 


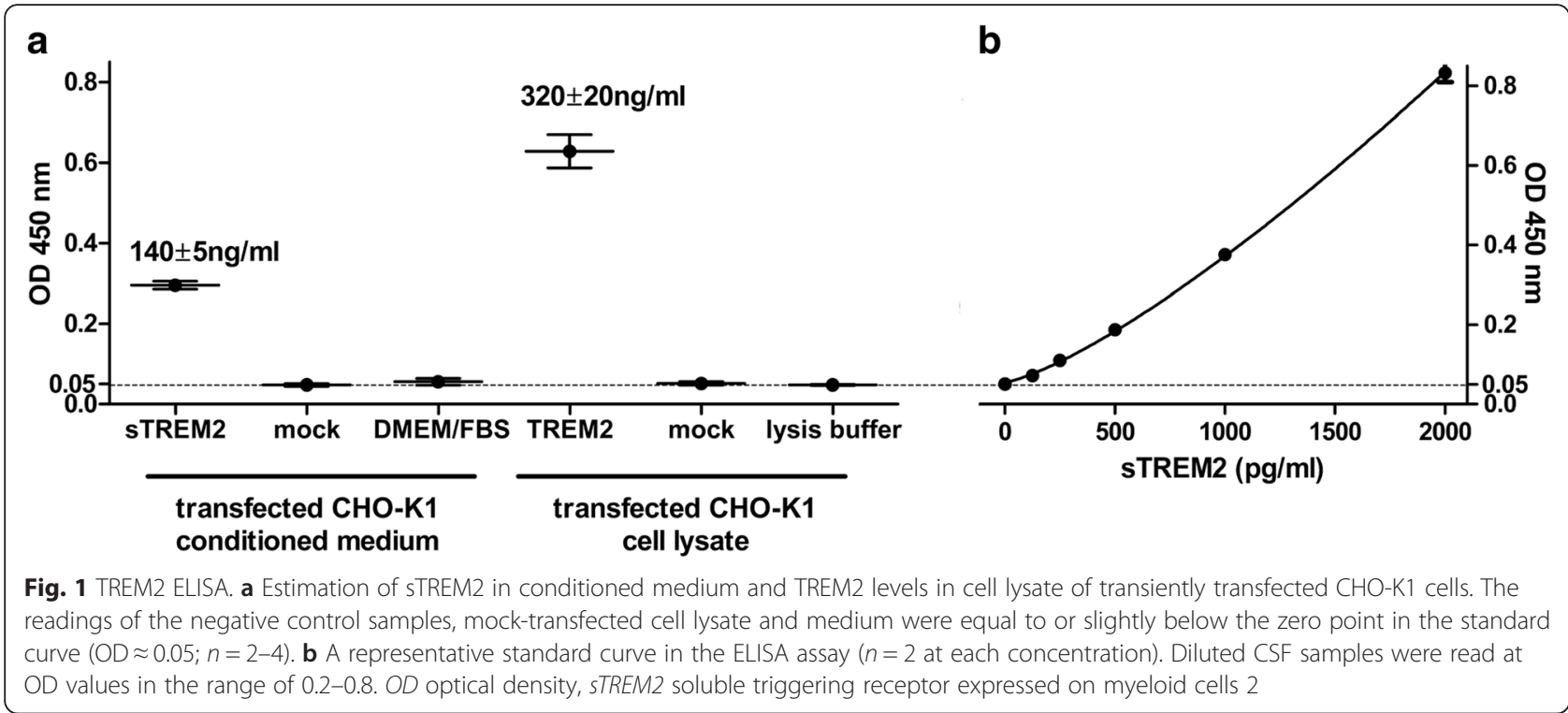

zero standard (Fig. $2 \mathrm{~b}$ ) and below the estimated ELISA detection limit (62.5 pg/ml, Fig. 1b). Repeated freeze-thaw cycles did not affect CSF sTREM2 levels in two random CSF samples (Additional file 1: Figure S2).

\section{CSF sTREM2 levels did not relate to clinical diagnosis or} degree of dementia

CSF STREM2 was measured in samples from a Swedish $\mathrm{AD} /$ control cohort and a Norwegian $\mathrm{AD} / \mathrm{MCI} /$ control cohort. The Norwegian cohort $(n=100)$ included controls $(n=50)$, MCI patients $(n=21)$ and AD patients $(n=29)$, and the Swedish cohort $(n=50)$ included controls $(n=25)$ and AD patients $(n=25)$. In line with the diagnosis, the $\mathrm{AD}$ patients had lower Mini-Mental State Examination (MMSE) scores and markedly reduced level of $A \beta 42$ and elevated levels of $T$ tau and P-tau in CSF as compared with controls in both cohorts. The more pronounced separation in CSF A $\beta 42$ levels between AD patients and controls in the Norwegian cohort was predicted because this cohort was diagnosed according to the revised NIA-AA criteria and controls were excluded if CSF A $\beta 42$ was below $550 \mathrm{pg} / \mathrm{ml}$. The groups in the Norwegian cohort were age matched, but the age range was wider among the controls. In the Swedish cohort, the controls were younger than the AD patients. The gender distribution was comparable in the control and MCI/AD groups of the Norwegian cohort. In the Swedish cohort, there were more women in both the control group and the $\mathrm{AD}$ group. The apolipoprotein $\mathrm{E}$ (ApoE) genotypes were similarly distributed in both

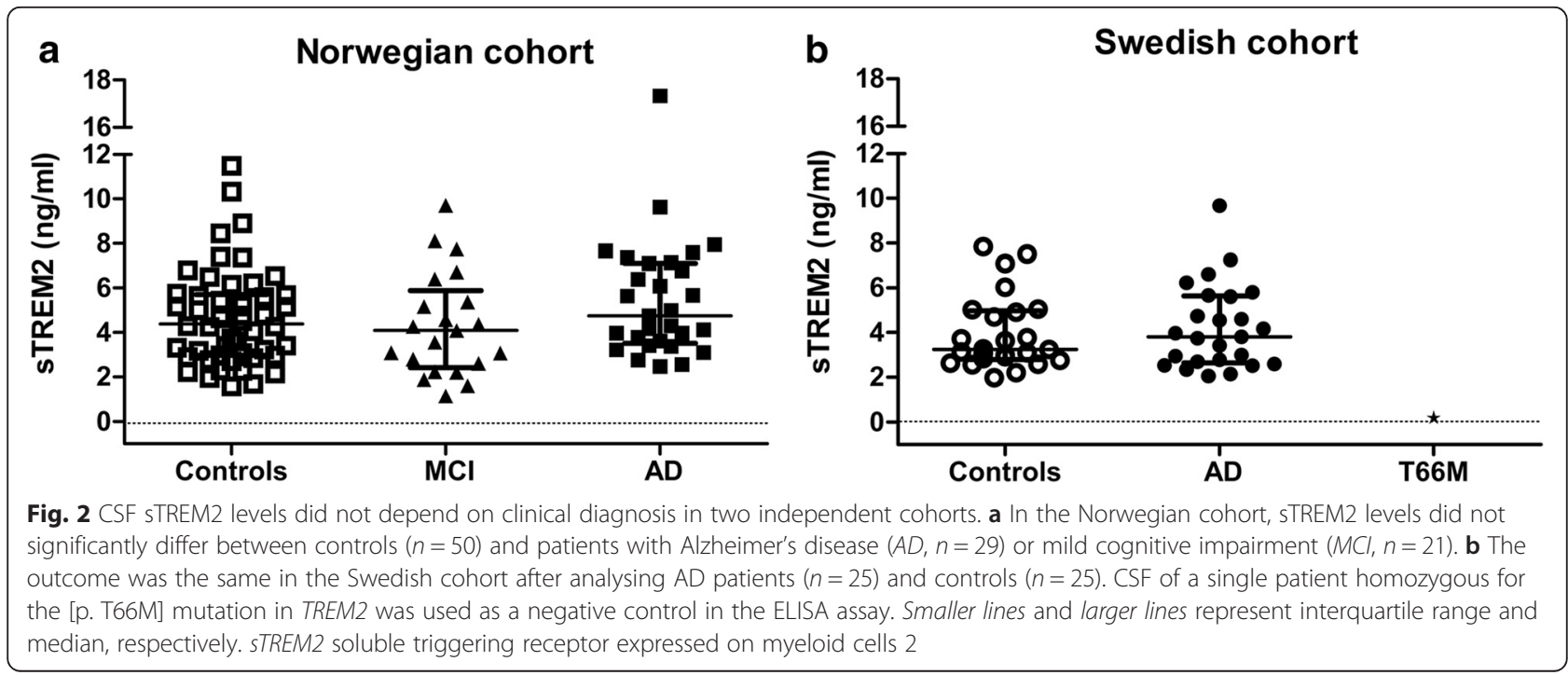


cohorts, with more E3/E3 carriers in the control groups and more $\mathrm{E} 3 / \mathrm{E} 4$ and $\mathrm{E} 4 / \mathrm{E} 4$ carriers in the disease groups (Table 1).

There were no statistically significant differences in the level of sTREM2 between the $\mathrm{MCI}, \mathrm{AD}$ and control groups in the Norwegian cohort (Fig. 2a and Table 1). The lack of difference in CSF sTREM2 levels between AD patients and controls was confirmed in the Swedish cohort (Fig. 2b and Table 1). All study groups displayed large variability (interquartile range/(median $\times 2) \approx 30-40 \%$ ) but sTREM2 levels tended to be slightly elevated in the $\mathrm{AD}$ group in both cohorts. When the cohorts were merged to increase statistical power, there was still no difference in STREM2 levels $(p=0.29)$ between AD patients $(n=54)$ and controls $(n=75)$. The level of sTREM2 and cognitive function (MMSE score) did not correlate even when the AD groups were merged (Spearman rho $=0.14$, $p=0.33, n=54)$. The lack of correlation between sTREM2 and MMSE score suggests that sTREM2 does not relate to cognitive ability in AD.

\section{CSF sTREM2 level correlated with age among controls}

Next, because age is the main risk factor for $\mathrm{AD}$, we questioned whether CSF sTREM2 levels related to normal aging. The analysis therefore focused on the control groups. As samples were assayed for sTREM2 in the same laboratory, the two cohorts were merged for this analysis. We found sTREM2 levels to increase with age in the control group, as demonstrated by a significant positive correlation (Spearman rho $=0.50 ; p<0.001 ; n=75$; Fig. 3). Interpolation of linear regression showed that sTREM 2 was $\approx 2.7 \mathrm{ng} / \mathrm{ml}$ at 50 years of age and $\approx 7.2 \mathrm{ng} /$ $\mathrm{ml}$ at 90 years of age (i.e. almost a threefold increase). When cohorts were analysed separately, age and sTREM2 levels correlated significantly in the Norwegian cohort

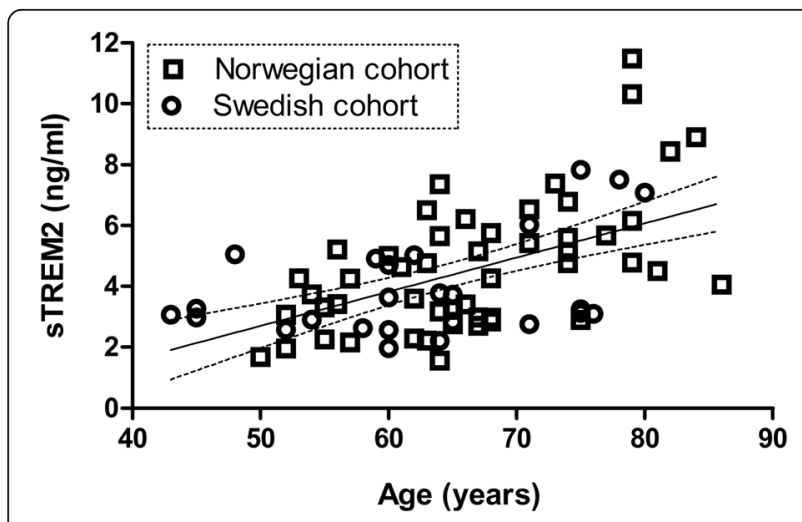

Fig. 3 CSF STREM2 levels correlated with age in the control groups. We found a significant positive correlation between age and STREM2 in the control groups (Spearman rho $=0.50 ; p<0.001 ; n=75$ ). The regression line (dotted lines representing the $95 \%$ confidence interval) is based on merged data for both cohorts. sTREM2 soluble triggering receptor expressed on myeloid cells 2
(Spearman rho $=0.53 ; p<0.001 ; n=50$ ), but the correlation did not reach statistical significance in the Swedish cohort (Spearman rho $=0.35 ; p=0.08 ; n=25$ ). In the $\mathrm{AD}$ groups we only found a positive correlation in the Swedish cohort (Spearman rho $=0.46 ; p=0.02 ; n=25$ ). The correlation was weaker, but still positive, when the $\mathrm{AD} / \mathrm{MCI}$ and control groups from both cohorts were merged (Spearman rho $=0.29 ; p<0.001 ; n=150$; Table 2).

\section{Relation between sTREM2 levels and AD CSF biomarker levels, $A \beta 42$ and P-tau/T-tau}

$\mathrm{AD}$ is a complex and pathologically rather heterogeneous disorder with substantial individual variability in the tolerance to neuropathological changes. We hypothesized that the levels of TREM2 would reflect pathogenic processes rather than clinical symptoms. Therefore, we examined the relation between sTREM2 levels and the AD CSF biomarkers $A \beta 42$, T-tau and P-tau in the larger Norwegian cohort in which the patients had been diagnosed according to the NIA-AA criteria.

A $\beta 42$ displayed a biphasic association pattern with sTREM2, with a distinction between $\mathrm{AD} / \mathrm{MCI}$ and controls (Fig. 4a). There was a positive correlation between sTREM2 and A 342 among all controls (Spearman rho = $0.35 ; p=0.01 ; n=50$ ) but not among AD/MCI patients (Table 3). Since A $\beta 42$ and sTREM2 levels seemed to depend on the diagnostic state, the material was stratified and further examined. Controls with high A $\beta 42(>700 \mathrm{pg} /$ $\mathrm{ml})$ were further analysed, since those with a lower $A \beta 42$ $(<700 \mathrm{pg} / \mathrm{ml})$ might already have amyloid pathology in the brain. The cut-off value of $700 \mathrm{pg} / \mathrm{ml}$ was supported by comparison between CSF A $\beta 42$ levels and amyloid-PET imaging in a separate group of Norwegian patients (Additional file 1: Figure S3). The correlation was stronger between CSF sTREM2 and A $\beta 42$ among controls with high A $\beta 42(>700 \mathrm{pg} / \mathrm{ml}$; Spearman rho $=0.44$; $p=0.002 ; n=46$; Fig. 5a and Table 3). CSF from many controls was also assayed with Meso Scale Discovery (MSD) for $A \beta 38, A \beta 40$ and $A \beta 42$. In controls with high A $342(>700 \mathrm{pg} / \mathrm{ml})$ there were significant positive correlations between sTREM2 and A 338 (Spearman rho $=0.51 ; p=0.004 ; n=31$ ), between sTREM2 and A $\beta 40$ (Spearman rho $=0.53 ; p=0.002 ; n=31$ ) and between STREM2 and MSD estimates of A $\beta 42$ (Spearman rho $=0.40 ; \mathrm{p}=0.03 ; n=31$ ) (Additional file 1: Figure S4 and Table 3). Estimation of A 442 with two independent techniques, Innotest ${ }^{\circ}$ and MSD correlated strongly (Spearman rho $=0.88 ; p<0.001 ; n=38$ ). MSD estimates of $A \beta 38$ and $A \beta 40$ in the same CSF sample also correlated very well (Spearman rho $=0.96 ; p<0.001 ; n=38$ ), while correlations between MSD analyses of $A \beta 42$ and $A \beta 38$ or $A \beta 40$ in the same samples were somewhat weaker (Spearman rho $=0.74 ; p<0.001 ; n=38$ ) (Additional file 1: Figure S5 and Table S1). 
Table 2 Correlation analyses of CSF STREM2 in relation to age in the Norwegian and the Swedish cohorts

\begin{tabular}{|c|c|c|c|c|c|c|c|c|c|c|c|}
\hline & \multicolumn{11}{|c|}{ Correlations between sTREM2 and age } \\
\hline & \multicolumn{5}{|c|}{ Norwegian cohort } & \multicolumn{3}{|c|}{ Swedish cohort } & \multicolumn{3}{|c|}{ Norwegian and Swedish cohort } \\
\hline & $\begin{array}{l}\text { All } \\
(n=100)\end{array}$ & $\begin{array}{l}\mathrm{AD} \\
(n=29)\end{array}$ & $\begin{array}{l}\mathrm{MCl} \\
(n=21)\end{array}$ & $\begin{array}{l}\text { Control } \\
(n=50)\end{array}$ & $\begin{array}{l}\mathrm{A} \beta 42>700 \\
(n=46)\end{array}$ & $\begin{array}{l}\text { All } \\
(n=50)\end{array}$ & $\begin{array}{l}\mathrm{AD} \\
(n=25)\end{array}$ & $\begin{array}{l}\text { Control } \\
(n=25)\end{array}$ & $\begin{array}{l}\text { All } \\
(n=150)\end{array}$ & $\begin{array}{l}\mathrm{AD} \\
(n=54)\end{array}$ & $\begin{array}{l}\text { Control } \\
(n=75)\end{array}$ \\
\hline \multirow[t]{2}{*}{ Age } & $r=0.34$ & $r=0.08$ & $r=0.03$ & $r=0.53$ & $r=0.56$ & $r=0.35$ & $r=0.46$ & $r=0.35$ & $r=0.29$ & $r=0.00$ & $r=0.50$ \\
\hline & $p<0.001$ & $p=0.69$ & $p=0.86$ & $p<0.001$ & $p<0.001$ & $p=0.01$ & $p=0.02$ & $p=0.08$ & $p<0.001$ & $p=1.00$ & $p<0.001$ \\
\hline
\end{tabular}

Correlation analyses of CSF sTREM2 in relation to age of $\mathrm{AD}, \mathrm{MCl}$ and control patients of the two cohorts

Correlations are presented as Spearman rho $(r)$ since most data not were normally distributed

A 42 amyloid beta 1-42, AD Alzheimer's disease, CSF cerebrospinal fluid, $M C I$ mild cognitive impairment, sTREM2 soluble triggering receptor expressed on myeloid cells 2

CSF sTREM2 correlated positively with the neurodegenerative biomarkers T-tau (Spearman rho $=0.57$; $p<0.001$; $n=50$; Fig. $4 \mathrm{~b}$ ) and P-tau in the control group alone (Spearman rho $=0.63 ; p<0.001 ; n=50$; Fig. $4 c$ ) and also when the control group was merged with the AD/MCI group. In the AD/MCI groups, the neurodegenerative biomarkers T-tau and P-tau did not relate to sTREM2. Similar plots and statistical calculations on data from the Swedish cohort only resulted in a positive correlation between sTREM2 and P-tau in controls (Spearman rho $=0.44$; $p=0.02 ; n=25$; Additional file 1: Figure S6 and Table 3).

sTREM2 levels correlated with age, $\mathrm{A} \beta 42, \mathrm{~A} \beta 40, \mathrm{~A} \beta 38$, T-tau and P-tau among controls with low likelihood of amyloid plaques, $(\mathrm{A} \beta 42>700 \mathrm{pg} / \mathrm{ml})$. Multiple linear regression was therefore performed using age and $A \beta 42$ (Fig. 5a), $A \beta 40$ or $A \beta 38$ as the explanatory variable for sTREM2 levels. The level of sTREM2 associated positively with age $(\beta 1=0.12 ; p<0.001)$ and $A \beta 42$ (Innotest $\left.{ }^{\circ}\right)$ ( $\beta 2=0.003, p=0.01, n=46$; Fig. $5 \mathrm{~b}$ and Additional file 1 : Table S2). There were also significant positive associations between sTREM2 levels and age and MSD estimates of $A \beta 38$ (age $\beta 1=0.08, p=0.02$; $A \beta 38 \beta 2=0.001$, $p=0.01, n=31$ ) and $\mathrm{A} \beta 40$ (age $\beta 1=0.07, p=0.02 ; \mathrm{A} \beta 40$ $\beta 2=0.0005, p=0.01, n=31$; Additional file 1: Figure S7). The corresponding analysis of sTREM2, age and the neurodegenerative marker P-tau in the same cohort showed a significant association with both explanatory variables: age $(\beta 1=0.11, \quad p=0.003)$ and P-tau $(\beta 1=0.04, p=0.02$, $n=46)$. The same multiple linear regression analysis of the entire control group (A $\beta 42$ Innotest ${ }^{\odot} / \mathrm{T}$-tau/P-tau $(n=50)$ or $\mathrm{A} \beta 38 / \mathrm{A} \beta 40 / \mathrm{A} \beta 42 \mathrm{MSD}(n=32))$ gave close to identical results (Additional file 1: Table S2).

\section{Discussion}

In the current study a new ELISA was developed and used to determine whether CSF sTREM2 related to AD, $\mathrm{AD}$ neurochemical biomarkers and cognitively healthy aging. A CSF sample from a patient homozygous for TREM2 [p. T66M] and cultured cells with or without TREM2 expression were used to validate the ELISA. We examined whether sTREM2 levels related to diagnosis and the AD CSF biomarkers A 342 , T-tau and P-tau, in two independent cohorts. While the Swedish cohort was diagnosed according to the NINCDS-ARDRA criteria [22], the Norwegian cohort was diagnosed according to the 2011 NIA-AA criteria with CSF biomarkers $[2,4]$. Naturally there was a more prominent difference in the CSF AD biomarker signature between controls and AD/ MCI in the Norwegian cohort. The levels of sTREM2, but not those of other biomarkers, were analysed in the same laboratory with a robust assay with low inter-plate and inter-day variability. We therefore considered it correct to pool the two cohorts when studying the relation between sTREM2 and aging. In contrast, each cohort

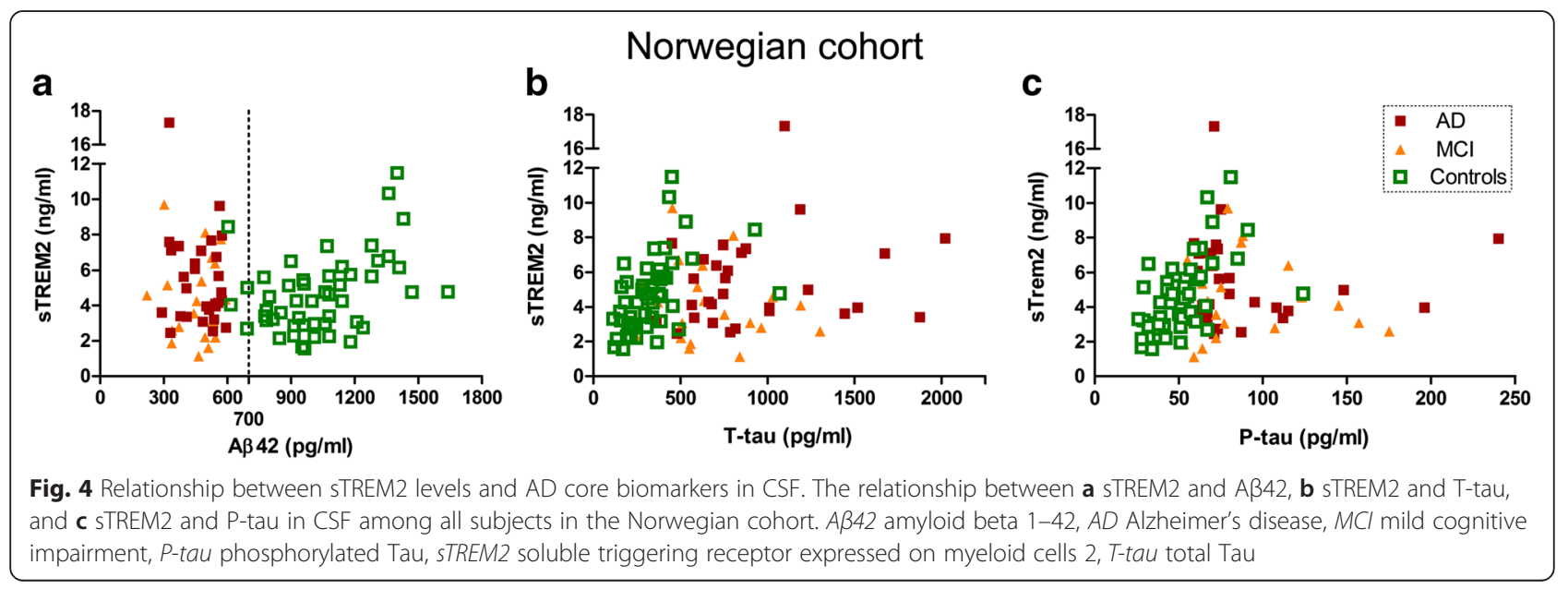


Table 3 Correlation analyses of CSF sTREM2 in relation to other AD CSF biomarkers in the Norwegian and Swedish cohorts

\begin{tabular}{|c|c|c|c|c|c|c|c|c|}
\hline & \multicolumn{8}{|c|}{ sTREM2 correlation } \\
\hline & \multicolumn{5}{|c|}{ Norwegian cohort } & \multicolumn{3}{|c|}{ Swedish cohort } \\
\hline & All $(n=100)$ & $\mathrm{AD}(n=29)$ & $\mathrm{MCl}(n=21)$ & Control $(n=50)$ & $\mathrm{A} \beta 42>700(n=46)$ & All $(n=50)$ & $\mathrm{AD}(n=25)$ & Control $(n=25)$ \\
\hline \multirow[t]{2}{*}{ T-tau } & $r=0.28$ & $r=0.22$ & $r=-0.06$ & $r=0.57$ & $r=0.63$ & $r=0.17$ & $r=0.05$ & $r=0.29$ \\
\hline & $p=0.005$ & $p=0.25$ & $p=0.81$ & $p<0.001$ & $p<0.001$ & $p=0.24$ & $p=0.82$ & $p=0.16$ \\
\hline \multirow[t]{2}{*}{ P-tau } & $r=0.33$ & $r=-0.04$ & $r=0.27$ & $r=0.63$ & $r=0.69$ & $r=0.17$ & $r=0.00$ & $r=0.44$ \\
\hline & $p=0.001$ & $p=0.85$ & $p=0.24$ & $p<0.001$ & $p<0.001$ & $p=0.23$ & $p=0.99$ & $p=0.02$ \\
\hline \multirow[t]{3}{*}{ Aß42 } & $r=0.04$ & $r=-0.05$ & $r=0.07$ & $r=0.35$ & $r=0.44$ & $r=0.09$ & $r=0.35$ & $r=0.06$ \\
\hline & $p=0.67$ & $p=0.79$ & $p=0.77$ & $p=0.01$ & $p=0.002$ & $p=0.55$ & $p=0.09$ & $p=0.79$ \\
\hline & & & & $n=32$ & $n=31$ & & & \\
\hline \multirow[t]{2}{*}{ Aß38 (MSD) } & & & & $r=0.49$ & $r=0.51$ & & & \\
\hline & & & & $p=0.005$ & $p=0.004$ & & & \\
\hline \multirow[t]{2}{*}{ Aß40 (MSD) } & & & & $r=0.51$ & $r=0.53$ & & & \\
\hline & & & & $p=0.003$ & $p=0.002$ & & & \\
\hline \multirow[t]{2}{*}{ Aß42 (MSD) } & & & & $r=0.37$ & $r=0.40$ & & & \\
\hline & & & & $p=0.04$ & $p=0.03$ & & & \\
\hline
\end{tabular}

Correlations between CSF sTREM2 levels and levels of T-tau, P-tau, A $342, A \beta 38$ MSD, A $A 40$ MSD and A 342 MSD in AD, MCl and control patients of the two cohorts. Correlations are presented as Spearman rho $(r)$ since most data not were normally distributed

A 42 amyloid beta 1-42, AD Alzheimer's disease, CSF cerebrospinal fluid, $M C l$ mild cognitive impairment, MSD Meso Scale Discovery, $P$-tau phosphorylated Tau, sTREM2 soluble triggering receptor expressed on myeloid cells 2, T-tau total Tau

was examined separately for correlations involving $A \beta 42$, T-tau or P-tau since those data were generated in different laboratories. We did not find any significant differences in CSF the level of sTREM2 between AD patients, MCI patients and controls. However in the control group, the level of sTREM2 correlated positively with aging.

The innate immune receptor TREM2 is predominantly expressed in microglia and other myeloid cells [12, 29]. sTREM2 was first found in human CSF and conditioned medium of dendritic cells [19]. It can be generated by sequential proteolysis of membrane-bound TREM2 [20], or by an alternatively splicing pathway resulting in a transcript lacking the transmembrane domain [30]. sTREM2 levels in CSF could depend on the synthesis rate of membrane-bound receptors in TREM2-expressing cells, largely microglia and peripherally derived macrophages, transport to the cell surface, shedding and degradation of the sTREM2 fragment. TREM2 gene expression is regulated by factors inducing myeloid cell differentiation [31]. TREM2 receptor recycling and shedding can also be regulated by cytokines (e.g. interleukin-13) [32]. The turnover of sTREM2 is unknown but other fragments generated by shedding (e.g. soluble A $\beta \mathrm{PP})$ have a short half-life $\left(t_{1 / 2} \approx\right.$ $4 \mathrm{~h}$ ) in the mouse brain [33].

We investigated whether sTREM2 levels altered with age, the most significant risk factor for $\mathrm{AD}$ and of importance
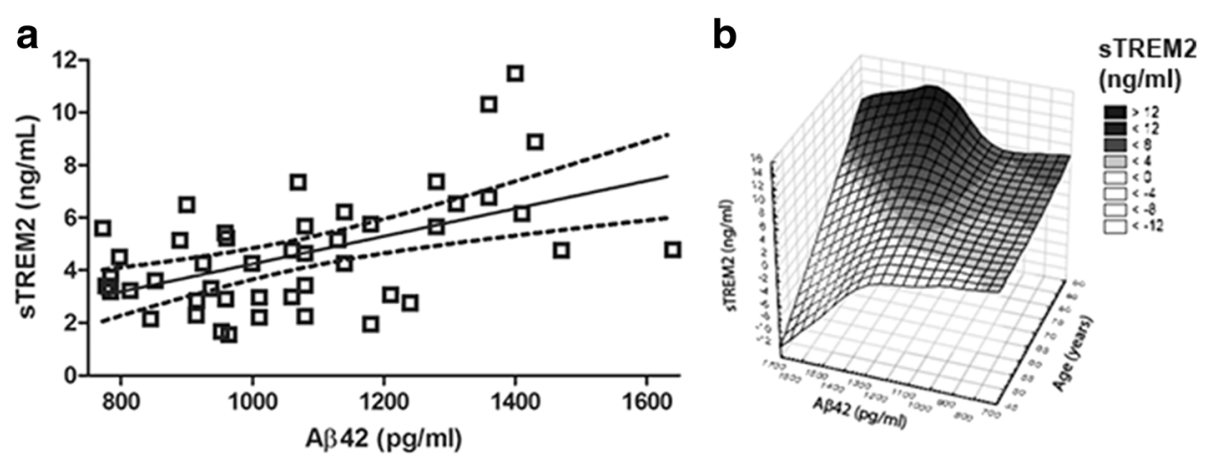

Fig. 5 Relationship between sTREM2 and A 42 and age in subjects with high CSF A 42 . Control subjects with high CSF A 342 (A $42>700 \mathrm{ng} / \mathrm{ml}$, $n=46$ ) were further analysed. a Relationship between sTREM2 and A 342 levels in CSF (Spearman rho $=0.44 ; p=0.002 ; n=46$ ), the solid line representing the linear regression line (dotted lines the $95 \%$ confidence interval). b Surface plot showing the relation between age ( $z$ axis), A $A 42(x$ axis) and sTREM2 levels (y axis). Aß42 amyloid beta 1-42, sTREM2 soluble triggering receptor expressed on myeloid cells 2 
to many neurodegenerative disorders. Aging is associated with gliosis, increased microglial activity [5] and astrocytosis in the brain [34]. In healthy controls we found a positive correlation between age and sTREM2 levels with almost a threefold increase from 50 to 90 years of age, suggesting that sTREM2 levels are related to the normal aging process. TREM2 mRNA was reported to increase by $50-100 \%$ from 50 to 90 years of age with healthy human aging in several brain regions [12]. In AD brain, the level of TREM2 protein was found to increase roughly $50 \%$ in the temporal cortex [35]. TREM2 mRNA expression was elevated to a similar extent and related to Braak staging [36]. Aging and AD thus enhanced TREM2 expression to a quantitatively similar extent in human brain. We found elevated CSF sTREM2 levels with aging but not with AD. Therefore mechanisms other than TREM2 expression presumably play a significant role in elevated sTREM2 levels with aging. We suggest that the agedependent increased CSF sTREM2 levels partly reflect enhanced microglial TREM2 expression but also induced shedding and/or reduced clearance. In the $\mathrm{AD} /$ MCI groups, pathogenic processes including neurodegeneration might overshadow the effect of aging on CSF sTREM2. Our data demonstrate the importance of age-matched study groups.

sTREM2 has been suggested as a possible biomarker of neuroinflammation, which is increasingly being recognized as an early event in AD [37]. Therefore it is conceivable that the level of CSF sTREM2 is increased in early stages of $\mathrm{AD}$, as it is in classical inflammatory conditions like multiple sclerosis [19]. However, the AD risk factor TREM2 [p. R47H] and frontotemporal dementia mutations [25] are most probably loss of function, resulting in reduced TREM2 cell surface localization and shedding [18]. If such changes are relevant to sporadic AD one would instead predict decreased CSF sTREM2. Indeed, in a previous study CSF sTREM2 was reduced in $\mathrm{AD}$ patients as compared with controls although intragroup variability was extensive [18]. Our study also showed large intra-group variability but sTREM2 levels did not differ between AD and controls. Hence we could not confirm reduced CSF sTREM2 in AD; instead, levels of sTREM2 tended to be higher in AD than in controls in both cohorts. We also included a group of MCI patients to see whether there were any differences in STREM2 relating to dementia. sTREM2 levels in MCI did not differ from either the control or the AD group. The different study outcome could depend on study populations or the assay format. Unlike the previous reports $[18,19]$, we measured absolute sTREM2 concentrations in the CSF samples. In the previous dementia study [18], in which CSF TREM2 was found to be reduced in $\mathrm{AD}$, the $\mathrm{AD}$ patients were approximately a decade older than the controls. This is similar to the
Swedish cohort in our report, but in contrast to the Norwegian cohort which was well matched for age. Agematching thus does not seem to explain the differences in study outcome, since we found a positive correlation between sTREM2 levels and age.

The role of TREM2 in AD pathology is only partly clear. TREM2 may have a neuroprotective function by regulating microglial/macrophage polarization [38] and serving as a phagocytic receptor [18, 39, 40]. TREM2 could thus serve to clear soluble $A \beta$-aggregates and other toxic debris, and control the inflammatory reactions elicited by the early AD pathology. However, the effects of TREM2 deficiency on amyloid plaque load in A $\beta P P$ transgenic mice are inconsistent [41-43]. We therefore compared the levels of sTREM2 with the AD neuropathological markers CSF A $\beta 42$, T-tau and P-tau. Interestingly we found a positive correlation between CSF sTREM2 and T-tau, P-tau and A $\beta 42$ in the control group. These correlations were not seen in the $\mathrm{AD}$ and MCI groups. The signature of low CSF A $\beta 42$ and high CSF T-tau/P-tau is established and enables prodromal AD diagnostics [44]. Several studies have found the neurodegenerative markers $\mathrm{T}$-tau and P-tau to increase almost threefold from 40 to 90 years with healthy aging $[24,45,46]$. Among aged subjects asymptomatic tauopathy is being reported [47]. Correlation of CSF sTREM2 levels with T-tau/P-tau could thus be an indirect effect of the age-dependent increase in $\mathrm{T}$-tau/P-tau.

The positive correlation between sTREM2 and A $\beta 42$ among controls is interesting because CSF A $\beta 42$ is ultimately reduced in association with amyloid deposition in $\mathrm{AD}$. CSF sTREM2 also correlated well with CSF measures of $A \beta 38$ and $A \beta 40$, and the different $A \beta$ measures correlated well with each other. The increased CSF $A \beta$ presumably reflects altered $A \beta$ metabolism, and not $A \beta 42$-selective changes associated with $A \beta$ deposition. In a previous study CSF A $\beta 42$ did not correlate with age among the cognitively healthy [24], while others reported a relation that best fit curve models of increasing and culminating $\mathrm{A} \beta 42[48,49]$ and $A \beta 40$ levels [49]. In the absence of amyloid deposits, an increased CSF-A $\beta$ peptide level probably reflects an imbalance between production and clearance of $A \beta$. There is evidence of decreased $A \beta$ clearance in aged individuals with sporadic AD [50]. Several mechanisms including agerelated changes to the vascular basement membrane and impaired drainage along the lymphatic drainage pathway are probably involved [51]. Amyloid formation depends on seeding; that is, the local concentration of $A \beta$ monomers must reach a critical threshold in order for fibril formation to begin [52]. Indeed, CSF A $\beta 42$ was shown to transiently increase by $20-30 \%$ in three inbred A $\beta P P$-transgenic models before it declined when amyloid plaques emerged [53]. We speculate that the positive correlation between CSF A $\beta$ peptides and CSF sTREM2 among controls reflects 
a very early pre-symptomatic stage of dementia. These findings are preliminary and need to be further examined in other cohorts and in familial AD.

\section{Conclusions}

A new sensitive TREM2 ELISA was used to determine CSF sTREM2 levels in cognitively healthy controls, and in patients with $\mathrm{AD}$ and $\mathrm{MCI}$. In the cognitively healthy, we found CSF sTREM2 levels to increase with age, the most important risk factor of AD. There was no statistically significant difference in sTREM2 levels between diagnostic states (healthy control, $\mathrm{AD}$ and $\mathrm{MCI}$ ), but sTREM2 levels correlated positively with the AD neuropathological CSF biomarkers $\mathrm{A} \beta 42$ and T-tau and P-tau in controls. Age should thus be considered when analysing sTREM2 in CSF. We speculate that increased CSF sTREM2 levels may reflect a very early microglial response to increased soluble $\mathrm{A} \beta 42$ in pre-symptomatic $\mathrm{AD}$ brain.

\section{Additional file}

Additional file 1: Figure S1 showing ELISA validation; Figure S2 showing freeze-thaw cycles; Figure S3 showing PET imaging versus CSF A 342 in patients; Figure 54 showing a scatter plot of CSF sTREM2 in

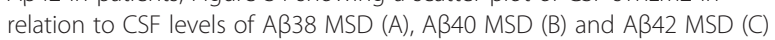
in the Norwegian $A D / M C l / c o n t r o l$ cohort; Figure $S 5$ showing a scatter plot of different CSF A 3 measures with Innotest ${ }^{\oplus}$ and MSD; Figure S6 showing a scatter plot of CSF sTREM2 in relation to levels of Aß42 (A), T-tau (B) and P-tau (C) in CSF in the Swedish AD/control cohort; Figure S7 showing a surface plot of CSF sTREM2 in relation to age and AB38 MSD (A) and in relation to age and A 40 MSD (B) in controls; Table S1 presenting correlations between different CSF A $\beta$ measures (A 42 Innotest ${ }^{\oplus}, A \beta 38$ MSD A 340 MSD and A 42 MSD); and Table S2 presenting correlation analyses between different CSF sTREM2 and age and biomarkers (A 342 Innotest ${ }^{\oplus}, A \beta 38$ MSD, A 40 MSD and Aß42 MSD, T-tau and P-tau). (PDF 647 kb)

\section{Abbreviations}

AD: Alzheimer's disease; ADAM: a disintegrin and metalloproteinase; ApoE: apolipoprotein $E ; A \beta$ : amyloid beta; $A \beta 42$ : amyloid beta 1-42; A $\beta P P$ : $A \beta$ precursor protein; $\mathrm{CHO}$ : Chinese hamster ovary; CSF: cerebrospinal fluid; CT: computed tomography; DAP12: DNAX activating protein 12; ELISA: enzyme-linked immunosorbent assay; MCl: mild cognitive impairment; MMSE: Mini-Mental State Examination; MRI: magnetic resonance imaging; MSD: Meso Scale Discovery; NFT: neurofibrillary tangle; NIA-AA: National Institute on Aging-Alzheimer's Association; NINCDS: National Institute of Neurological and Communicative Disorders and Stroke; PET: positron emission tomography; P-tau: phosphorylated Tau; RT: room temperature; sTREM2: soluble triggering receptor expressed on myeloid cells 2; TREM2: triggering receptor expressed on myeloid cells 2; T-tau: total Tau.

\section{Competing interests}

The authors declare that they have no competing interests.

\section{Authors' contributions}

$\mathrm{KH}$ designed the study and performed the experiments, analysed data, and wrote the first draft and the final manuscript. ISA structured the data bank, selected clinical samples and analysed data. VÅ developed analytical tools and performed experiments. $\mathrm{OH}$ and LM supervised clinical data bank collection and gave clinical advice. TF supervised clinical studies and contributed with critical advice. LNGN conceived of the study, supervised the project and wrote the final manuscript. All authors read, contributed to critical revisions and approved the final manuscript.

\section{Acknowledgements}

This work was supported by funding from University of Oslo, Anders Jahres stiftelse, Helse Sør-Øst and Civitan Foundation (to LNGN), by a JPND grant (APGeM; to TF and LNGN) and by grants from the Norwegian Research Council (NASATS, DDl; to TF) and Vetenskapsrådet (2013-2923; to OH). Professor John Hardy, Dr Ebba Lohmann and Jamie Toombs are greatly acknowledged for facilitating a generous gift of the TREM2 [p.T66M] CSF sample. The authors thank the Leonard Wolfson Experimental Neurology Centre, University College of London for supporting the collection of CSF samples at the biobank.

\section{Author details}

${ }^{1}$ Department of Pharmacology, University of Oslo and Oslo University Hospital, P.b. 1057, Blindern NO-0316, Oslo, Norway. ${ }^{2}$ Department of Neurology, Akershus University Hospital, Lørenskog, Norway. ${ }^{3}$ Department of Neurology, Faculty Division, Akershus University Hospital, University of Oslo, Lørenskog, Norway. ${ }^{4}$ Department of Clinical Sciences Malmö, Memory Clinic, Clinical Memory Research Unit, Lund University, Malmö, Sweden.

Received: 9 December 2015 Accepted: 30 March 2016

Published online: 27 April 2016

\section{References}

1. Hyman BT, Phelps CH, Beach TG, Bigio EH, Cairns NJ, Carrillo MC, et al. National Institute on Aging-Alzheimer's Association guidelines for the neuropathologic assessment of Alzheimer's disease. Alzheimers Dement. 2012;8:1-13.

2. Albert MS, DeKosky ST, Dickson D, Dubois B, Feldman HH, Fox NC, et al. The diagnosis of mild cognitive impairment due to Alzheimer's disease: recommendations from the National Institute on Aging-Alzheimer's Association workgroups on diagnostic guidelines for Alzheimer's disease. Alzheimers Dement. 2011;7:270-9.

3. Jack Jr CR, Albert MS, Knopman DS, McKhann GM, Sperling RA, Carrillo MC, et al. Introduction to the recommendations from the National Institute on Aging-Alzheimer's Association workgroups on diagnostic guidelines for Alzheimer's disease. Alzheimers Dement. 2011;7:257-62.

4. McKhann GM, Knopman DS, Chertkow H, Hyman BT, Jack Jr CR, Kawas CH, et al. The diagnosis of dementia due to Alzheimer's disease: recommendations from the National Institute on Aging-Alzheimer's Association workgroups on diagnostic guidelines for Alzheimer's disease. Alzheimers Dement. 2011;7:263-9.

5. Heneka MT, Kummer MP, Latz E. Innate immune activation in neurodegenerative disease. Nat Rev Immunol. 2014;14:463-77.

6. Zimmer ER, Leuzy A, Benedet AL, Breitner J, Gauthier S, Rosa-Neto P. Tracking neuroinflammation in Alzheimer's disease: the role of positron emission tomography imaging. J Neuroinflammation. 2014;11:120.

7. Fagan AM, Perrin RJ. Upcoming candidate cerebrospinal fluid biomarkers of Alzheimer's disease. Biomark Med. 2012;6:455-76.

8. Craig-Schapiro R, Perrin RJ, Roe CM, Xiong C, Carter D, Cairns NJ, et al. YKL-40: a novel prognostic fluid biomarker for preclinical Alzheimer's disease. Biol Psychiatry. 2010;68:903-12.

9. Hollingworth P, Harold D, Sims R, Gerrish A, Lambert JC, Carrasquillo MM, et al. Common variants at ABCA7, MS4A6A/MS4A4E, EPHA1, CD33 and CD2AP are associated with Alzheimer's disease. Nat Genet. 2011;43:429-35.

10. Lambert JC, Heath S, Even G, Campion D, Sleegers K, Hiltunen M, et al. Genome-wide association study identifies variants at CLU and CR1 associated with Alzheimer's disease. Nat Genet. 2009;41:1094-9.

11. Lambert JC, Ibrahim-Verbaas CA, Harold D, Naj AC, Sims R, Bellenguez C, et al. Meta-analysis of 74,046 individuals identifies 11 new susceptibility loci for Alzheimer's disease. Nat Genet. 2013;45:1452-8.

12. Forabosco P, Ramasamy A, Trabzuni D, Walker R, Smith C, Bras J, et al. Insights into TREM2 biology by network analysis of human brain gene expression data. Neurobiol Aging. 2013;34:2699-714.

13. Hickman SE, El KJ. TREM2 and the neuroimmunology of Alzheimer's disease. Biochem Pharmacol. 2014;88:495-8.

14. Guerreiro R, Wojtas A, Bras J, Carrasquillo M, Rogaeva E, Majounie E, et al. TREM2 variants in Alzheimer's disease. N Engl J Med. 2013;368:117-27.

15. Jonsson T, Stefansson H, Steinberg S, Jonsdottir I, Jonsson PV, Snaedal J, et al. Variant of TREM2 associated with the risk of Alzheimer's disease. N Engl J Med. 2013;368:107-16.

16. Zhang B, Gaiteri C, Bodea LG, Wang Z, McElwee J, Podtelezhnikov AA, et al. Integrated systems approach identifies genetic nodes and networks in late-onset Alzheimer's disease. Cell. 2013;153:707-20. 
17. Frank S, Burbach GJ, Bonin M, Walter M, Streit W, Bechmann I, et al. TREM2 is upregulated in amyloid plaque-associated microglia in aged APP23 transgenic mice. Glia. 2008;56:1438-47.

18. Kleinberger G, Yamanishi Y, Suarez-Calvet M, Czirr E, Lohmann E, Cuyvers E, et al. TREM2 mutations implicated in neurodegeneration impair cell surface transport and phagocytosis. Sci Transl Med. 2014;6:243ra86.

19. Piccio L, Buonsanti C, Cella M, Tassi I, Schmidt RE, Fenoglio C, et al. Identification of soluble TREM-2 in the cerebrospinal fluid and its association with multiple sclerosis and CNS inflammation. Brain. 2008;131(Pt 11):3081-91.

20. Wunderlich P, Glebov K, Kemmerling N, Tien NT, Neumann H, Walter J. Sequential proteolytic processing of the triggering receptor expressed on myeloid cells-2 (TREM2) protein by ectodomain shedding and gammasecretase-dependent intramembranous cleavage. J Biol Chem. 2013;288: 33027-36.

21. McKhann G. Diagnostics and statistical manual of mental disorders. Arlington, VA: American Psychiatric Association; 1987.

22. McKhann G, Drachman D, Folstein M, Katzman R, Price D, Stadlan EM Clinical diagnosis of Alzheimer's disease: report of the NINCDS-ADRDA Work Group under the auspices of Department of Health and Human Services Task Force on Alzheimer's Disease. Neurology. 1984;34:939-44.

23. Mulder C, Verwey NA, van der Flier WM, Bouwman FH, Kok A, van Elk EJ, et al. Amyloid-beta(1-42), total tau, and phosphorylated tau as cerebrospinal fluid biomarkers for the diagnosis of Alzheimer disease. Clin Chem. 2010;56:248-53.

24. Sjögren $M$, Vanderstichele $H$, Agren $H$, Zachrisson $O$, Edsbagge M, Wikkelso C, et al. Tau and Abeta42 in cerebrospinal fluid from healthy adults 21-93 years of age: establishment of reference values. Clin Chem. 2001;47:1776-81.

25. Guerreiro RJ, Lohmann E, Bras JM, Gibbs JR, Rohrer JD, Gurunlian N, et al. Using exome sequencing to reveal mutations in TREM2 presenting as a frontotemporal dementia-like syndrome without bone involvement. JAMA Neurol. 2013;70:78-84.

26. Blennow K, Wallin A, Agren H, Spenger C, Siegfried J, Vanmechelen E. Tau protein in cerebrospinal fluid: a biochemical marker for axonal degeneration in Alzheimer disease? Mol Chem Neuropathol. 1995;26:231-45.

27. Vanmechelen $\mathrm{E}$, Vanderstichele $\mathrm{H}$, Davidsson $\mathrm{P}$, Van KE, Van Der Perre B, Sjogren M, et al. Quantification of tau phosphorylated at threonine 181 in human cerebrospinal fluid: a sandwich ELISA with a synthetic phosphopeptide for standardization. Neurosci Lett. 2000;285:49-52.

28. Vanderstichele $H$, Van KE, Hesse C, Davidsson P, Buyse MA, Andreasen N, et al. Standardization of measurement of beta-amyloid(1-42) in cerebrospinal fluid and plasma. Amyloid. 2000;7:245-58.

29. Hickman SE, Kingery ND, Ohsumi TK, Borowsky ML, Wang LC, Means TK, et al. The microglial sensome revealed by direct RNA sequencing. Nat Neurosci. 2013;16:1896-905.

30. Jin SC, Benitez BA, Karch CM, Cooper B, Skorupa T, Carrell D, et al. Coding variants in TREM2 increase risk for Alzheimer's disease. Hum Mol Genet. 2014;23:5838-46

31. Bouchon A, Hernandez-Munain C, Cella M, Colonna M. A DAP12-mediated pathway regulates expression of CC chemokine receptor 7 and maturation of human dendritic cells. J Exp Med. 2001;194(8):1111-22.

32. Wu K, Byers DE, Jin X, Agapov E, Alexander-Brett J, Patel AC, et al. TREM-2 promotes macrophage survival and lung disease after respiratory viral infection. J Exp Med. 2015;212:681-97.

33. Morales-Corraliza J, Mazzella MJ, Berger JD, Diaz NS, Choi JH, Levy E, et al. In vivo turnover of tau and APP metabolites in the brains of wild-type and Tg2576 mice: greater stability of sAPP in the beta-amyloid depositing mice. PLoS One. 2009;4:e7134.

34. Nichols NR, Day JR, Laping NJ, Johnson SA, Finch CE. GFAP mRNA increases with age in rat and human brain. Neurobiol Aging. 1993;14:421-9.

35. Lue LF, Schmitz CT, Sorrano G, Sue LI, Beach TG, Walker DG. TREM2 protein expression changes correlate with Alzheimer's disease neurodegenerative pathologies in postmortem temporal cortices. Brain Pathol. 2015;25:469-80.

36. Martiskainen $H$, Viswanathan J, Nykanen NP, Kurki M, Helisalmi S, Natunen T, et al. Transcriptomics and mechanistic elucidation of Alzheimer's disease risk genes in the brain and in vitro models. Neurobiol Aging. 2015;36:1221-8.

37. Rodriguez-Vieitez E, Saint-Aubert L, Carter SF, Almkvist O, Farid K, Scholl M et al. Diverging longitudinal changes in astrocytosis and amyloid PET in autosomal dominant Alzheimer's disease. Brain. 2016;139:922-36.

38. Kawabori M, Kacimi R, Kauppinen T, Calosing C, Kim JY, Hsieh CL, et al. Triggering receptor expressed on myeloid cells 2 (TREM2) deficiency attenuates phagocytic activities of microglia and exacerbates ischemic damage in experimental stroke. J Neurosci. 2015;35:3384-96.
39. N'Diaye EN, Branda CS, Branda SS, Nevarez L, Colonna M, Lowell C, et al. TREM-2 (triggering receptor expressed on myeloid cells 2 ) is a phagocytic receptor for bacteria. J Cell Biol. 2009;184:215-23.

40. Hsieh CL, Koike M, Spusta SC, Niemi EC, Yenari M, Nakamura MC, et al. A role for TREM2 ligands in the phagocytosis of apoptotic neuronal cells by microglia. J Neurochem. 2009;109:1144-56.

41. Jay TR, Miller CM, Cheng PJ, Graham LC, Bemiller S, Broihier ML, et al. TREM2 deficiency eliminates TREM2+ inflammatory macrophages and ameliorates pathology in Alzheimer's disease mouse models. J Exp Med. 2015;212:287-95.

42. Ulrich JD, Finn MB, Wang Y, Shen A, Mahan TE, Jiang H, et al. Altered microglial response to Abeta plaques in APPPS1-21 mice heterozygous for TREM2. Mol Neurodegener. 2014;9:20.

43. Wang Y, Cella M, Mallinson K, Ulrich JD, Young KL, Robinette ML, et al. TREM2 lipid sensing sustains the microglial response in an Alzheimer's disease model. Cell. 2015:160:1061-71.

44. Hansson O, Zetterberg H, Buchhave P, Londos E, Blennow K, Minthon L. Association between CSF biomarkers and incipient Alzheimer's disease in patients with mild cognitive impairment: a follow-up study. Lancet Neurol. 2006;5:228-34.

45. Morris JC, Roe CM, Xiong C, Fagan AM, Goate AM, Holtzman DM, et al. APOE predicts amyloid-beta but not tau Alzheimer pathology in cognitively normal aging. Ann Neurol. 2010;67:122-31.

46. Paternico D, Galluzzi S, Drago V, Bocchio-Chiavetto L, Zanardini R, Pedrini L, et al. Cerebrospinal fluid markers for Alzheimer's disease in a cognitively healthy cohort of young and old adults. Alzheimers Dement. 2012;8:520-7.

47. Crary JF, Trojanowski JQ, Schneider JA, Abisambra JF, Abner EL, Alafuzoff I, et al. Primary age-related tauopathy (PART): a common pathology associated with human aging. Acta Neuropathol. 2014;128:755-66.

48. Burkhard PR, Fournier R, Mermillod B, Krause KH, Bouras C, Irminger I. Cerebrospinal fluid tau and Abeta42 concentrations in healthy subjects: delineation of reference intervals and their limitations. Clin Chem Lab Med. 2004:42:396-407.

49. Shoji M, Kanai M. Cerebrospinal fluid Abeta40 and Abeta42: natural course and clinical usefulness. J Alzheimers Dis. 2001;3:313-21.

50. Mawuenyega KG, Sigurdson W, Ovod V, Munsell L, Kasten T, Morris JC, et al. Decreased clearance of CNS beta-amyloid in Alzheimer's disease. Science. 2010;330:1774.

51. Hawkes CA, Hartig W, Kacza J, Schliebs R, Weller RO, Nicoll JA, et al. Perivascular drainage of solutes is impaired in the ageing mouse brain and in the presence of cerebral amyloid angiopathy. Acta Neuropathol. 2011;121:431-43.

52. Jarrett JT, Lansbury Jr PT. Seeding 'one-dimensional crystallization' of amyloid: a pathogenic mechanism in Alzheimer's disease and scrapie? Cell. 1993:73:1055-8.

53. Maia LF, Kaeser SA, Reichwald J, Lambert M, Obermuller U, Schelle J, et al. Increased CSF Abeta during the very early phase of cerebral Abeta deposition in mouse models. EMBO Mol Med. 2015;7:895-903.

\section{Submit your next manuscript to BioMed Central and we will help you at every step:}

- We accept pre-submission inquiries

- Our selector tool helps you to find the most relevant journal

- We provide round the clock customer support

- Convenient online submission

- Thorough peer review

- Inclusion in PubMed and all major indexing services

- Maximum visibility for your research

Submit your manuscript at www.biomedcentral.com/submit 